\title{
Endurance Ablation Facilitates Long-lasting Circumferential Pulmonary Vein Isolation and Better Clinical Outcomes in Patients with Paroxysmal Atrial Fibrillation
}

Hee Tae Yu, MD, PhD1; Junbeom Park, MD, PhD2; Tae-Hoon Kim, MD1; Jae-Sun Uhm, MD, PhD1; JongYoun Kim, MD, PhD1; Boyoung Joung, MD, PhD1; Moon-Hyoung Lee, MD, PhD1; Hui-Nam Pak, MD, $\mathrm{PhD}^{1}$

'Yonsei University Health System, Seoul, Republic of Korea; 2Ewha Womans University, Seoul, Republic of Korea The first two authors contributed equally to this work.

Received: July 28, 2017

Revision Received: August 20, 2017 Accepted: August 31, 2017

Correspondence: Hui-Nam Pak, MD, PhD Yonsei University Health System, 50 Yonsei-ro, Seodaemun-gu, Seoul 03722, Republic of Korea Tel: +82-2-2228-8459 Fax: +82-2-393-2041 E-mail: hnpak@yuhs.ac

Copyright (C) 2017 The Official Journal of Korean Heart Rhythm Society Editorial Board and MMK Communications Limited

\begin{abstract}
Background: Recurrence of atrial fibrillation (AF) after radiofrequency catheter ablation was found to be related to the reconnection of circumferential pulmonary vein isolation (CPVI). We hypothesized that endurance ablation with moderately increased radiofrequency (RF) power would facilitate long-lasting $\mathrm{CPVI}$ and better clinical outcomes. Methods: We included 508 patients with paroxysmal AF $(76.0 \%$ men; mean age, 56.2 \pm 9.5 years) who underwent CPVI alone. We compared endurance ablation group (ENDU-abl; n=254, 30 s ablation at each point with $35 \mathrm{~W}$ on anterior pulmonary veins (PVs) antrum and 25-30 W on posterior left atrium [LA]) with age-, sex-, and follow-up duration-matched conventional ablation group (CONVabl; $n=254$, elimination of PV potential [PVP] with $25 \mathrm{~W}$ ).
\end{abstract}

Results: 1. Total procedure time $(p<0.001)$ and ablation time $(p<0.001)$ were significantly longer, and major complication rate was comparable $(p=0.088)$ between ENDU-abl and CONV-abl groups; 2. At 3-month follow-up, heart rate variability, high-frequency (HF) domain $(p=0.008)$ and low-frequency (LF)/HF ratio $(p<0.001)$ were significantly lower, while LA reverse remodeling was more significant at 1 -year follow-up echocardiogram $(p=0.013)$ in ENDU-abl group; 3. During 37.2 \pm 17.5 months of follow-up, the clinical recurrence of AF was significantly lower in ENDU-abl group (19.3\% vs. $35.4 \%$, log-rank $p<0.001)$; 4 . Among 38 patients who underwent redo-ablation at $15.1 \pm 12.1$ months, reconnected PVPs were found in $4 / 11$ patients (36.4\%) from ENDU-abl group and in $26 / 27$ patients $(96.3 \%)$ from CONV-abl group $(p<0.001)$.

Conclusion: Despite longer procedure time and relatively higher complication risk, endurance ablation with moderately increased RF power facilitates long-lasting CPVI, more significant autonomic neural remodeling and LA reverse remodeling, and better clinical outcome than conventional ablation in patients with paroxysmal AF.

Key Words: - Atrial Fibrillation - Catheter Ablation - Endurance Ablation -Heart Rate Variability 


\section{Introduction}

Radiofrequency catheter ablation (RFCA) is known to be an effective rhythm control strategy for anti-arrhythmic drug resistant atrial fibrillation (AF). Several different approaches are available for AF catheter ablation ${ }^{1,2}$; however, ablation around the pulmonary veins $(\mathrm{PV})$ to disconnect electrical conduction between the left atrium (LA) and the PV is the cornerstone of the procedure. Therefore, secure and long-lasting circumferential PV isolation (CPVI) promotes good clinical outcomes, and PV reconnection plays a major role in the clinical recurrence of $\mathrm{AF}$ after catheter ablation. ${ }^{3}$ Antiarrhythmic effects of CPVI are related not only to generating conduction blocks of PV triggers, but also to critical mass reduction ${ }^{4}$ or cardiac autonomic nerve modulation. ${ }^{5}$ Because the ganglionated plexi of cardiac autonomic nerves are located mainly along the epicardial aspect of the PV antrum, transmural lesion formation is important during CPVI. However, endocardial catheter ablation technology has limitations, such as incomplete transmural lesions, recovery of conduction, and difficulty in cardiac autonomic nerve modification. Therefore, adequate energy applied for an appropriate duration, proper tissue contact, and stable catheter location are important factors for the generation of homogeneous transmural CPVI lesions. In contrast, excess atrial damage due to excessive power or prolonged duration of ablation may induce collateral damage or may sometimes have proarrhythmic effect. ${ }^{6}$ Although the development of the irrigated tip catheter and contact force monitoring technology has enabled a relatively effective and safe procedure, ${ }^{7} \mathrm{AF}$ ablation remains operator dependent. Therefore, we hypothesized that moderately increased radiofrequency $(\mathrm{RF})$ power with endurance tissue contact may guarantee long-lasting CPVI and better clinical outcomes than conventional CPVI, which only aims to eliminate PV potential (PVP). The purpose of this study was to compare the endurance ablation (ENDU-abl: $30 \mathrm{~s}$ ablation at each point) with moderately increased RF power $(35 \mathrm{~W})$ with $25 \mathrm{~W}$ conventional ablation (CONV-abl: elimination of PVP only) in terms of complication rates, clinical outcomes, heart rate variability (HRV) at three months after the procedure, and mapping findings at redo-ablation procedures.

\section{Methods}

\section{Study population}

The study protocol adhered to the guidelines of the Declaration of Helsinki and was approved by the Institutional Review Board of Yonsei University Health System. All patients gave written informed consent before enrollment. From 1,989 consecutive patients included in the Yonsei AF Ablation Cohort (March 2009 to April 2016), we identified 999 patients with paroxysmal AF (PAF), who underwent only CPVI without additional LA ablation. From them, we included 254 patients in the ENDUabl group and compared them with 254 age-, sex-, and follow-up duration-matched patients from the CONV-abl group. The study's exclusion criteria were as 1) persistent or permanent AF;2) valvular AF; 3) LA ablation other than CPVI; 4) prior cardiac surgery or catheter ablation. All antiarrhythmic drugs were discontinued for a period corresponding to at least five half-lives. Anticoagulation therapy was maintained prior to catheter ablation.

\section{Echocardiogram and CT combined with electroanatom- ical LA mapping}

Trans-thoracic echocardiography (TTE) was performed in all patients prior to RFCA. Chamber size, transmitral flow velocity, and tissue Doppler images of the mitral annular septal area were acquired according to the American Society of Echocardiography guidelines. ${ }^{8}$ Three-dimensional (3D) spiral computerized tomography (CT) scans (64 Channel, Light Speed Volume CT, Philips, Brilliance 63, the Netherlands) were performed to define the PV anatomy and to analyze anatomical compartments of the LA. A 3D electroanatomical map (NavX, St. Jude Medical Inc., Minnetonka, MN, USA; CARTO 3, Johnson \& Johnson Inc., Diamond Bar, CA, USA) was generated using a circular PV mapping catheter (Lasso; Biosense-Webster Inc., Diamond Bar, CA, USA). NavX system-generated 3D geometry of the LA and of the PV was merged with the corresponding $3 \mathrm{D}$ spiral CT images. 
RFCA

An open irrigated tip, 3.5-mm-tip deflectable catheter (Thermocool, Johnson \& Johnson Inc., Diamond Bar, CA, USA; Coolflex, St. Jude Medical Inc., Minnetonka, MN, USA) was used for RFCA. An ENDU-abl was performed by maintaining the ablation catheter at each target point for $30 \mathrm{~s}$, regardless of electrogram voltage abatement, using $35 \mathrm{~W} R F$ power on the anterior aspects of PV antrum. However, we reduced the RF power to $25-30 \mathrm{~W}$ at the LA posterior wall and moved the ablation point before reaching $30 \mathrm{~s}$, as soon as voltage reduction was detected on the electrogram or the patient complained of pain. We compared 254 patients from ENDU-abl group with 254 age-, sex-, and follow-up duration-matched patients from CONVabl group. A CONV-abl was performed with $25 \mathrm{~W}$ RF power on the anterior and posterior aspects of $\mathrm{PV}$ antrum. The aim of the CONV-abl was only to eliminate PVPs; therefore, movement of the ablation point was guided by watching for the reduction of the electrogram voltage without waiting for $30 \mathrm{~s}$ (Figure 1). The endpoint of ablation at each site was an $>80 \%$ decrease in the local electrogram voltage amplitude. However, the endpoint of the overall procedure was the same in both groups: 1) CPVI with or without dissociated PVPs confirmed using bidirectional block, 2) bidirectional cavotricuspid isthmus block, and 3) no immediate recurrence of AF within 10 min after cardioversion with an isoproterenol infusion $(5 \mu \mathrm{g} / \mathrm{min})$.

\section{Post-ablation management and follow-up}

All patients were followed-up and antiarrhythmic drugs were discontinued after RFCA. Patients visited an outpatient clinic regularly at one, three, six, and 12 months after the procedure and subsequently every six months, or whenever symptoms occurred after RFCA. An electrocardiogram (ECG) was performed in all patients at every visit and a 24- or 48-hour Holter recording and/ or event recording was also performed at three, six, and every six months after the procedure, according to the 2012 HRS/EHRA/ ECAS Expert Consensus Statement guidelines. ${ }^{9}$ However,

\section{A 25 W Conventional ablation}

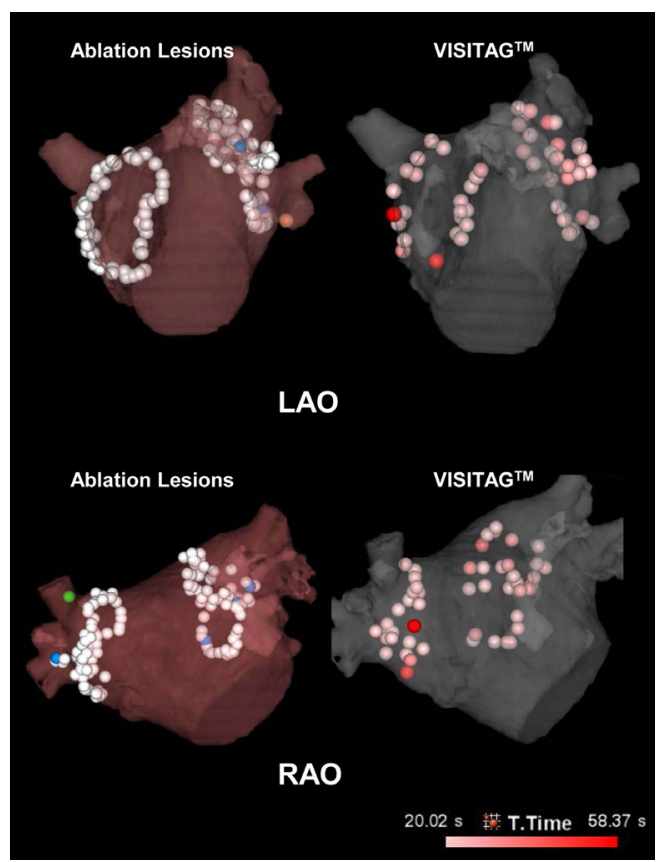

B 35 W Endurance ablation

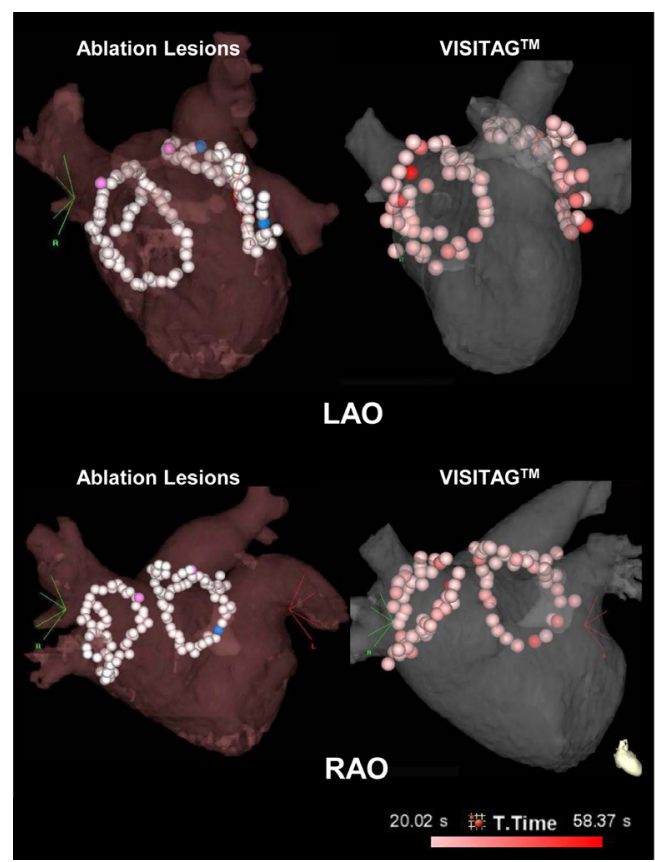

Figure 1. Representative CPVI of conventional ablation (A) and endurance ablation (B) visualized using VISITAGTM Module image (Johnson \& Johnson Inc., Diamond Bar, CA, USA). The colors of the spheres represent the duration of ablation (from white to red) in each location (diameter range $\leq 5 \mathrm{~mm}$ ). The diameter of each sphere was $2 \mathrm{~mm}$, and the gaps without spheres indicate the areas with ablation duration $<20 \mathrm{~s}$ (color scale bar) around the PV.

$\mathrm{CPVI}$, circumferential pulmonary vein isolation; $\mathrm{LAO}$, left anterior oblique; $\mathrm{PV}$, pulmonary vein; $\mathrm{RAO}$, right anterior oblique 
whenever patients reported palpitations, Holter monitor or event monitor recordings evaluated for possible recurrence of arrhythmia. We defined a recurrence of AF as any episode of $\mathrm{AF}$ or atrial tachycardia lasting for at least $30 \mathrm{~s}$ in duration. ${ }^{9}$ Any ECG documentation of AF recurrence after three months was diagnosed as clinical recurrence, 9 and antiarrhythmic medication was prescribed. We recommended redo-ablation for patients who showed clinical recurrence of AF. At the time of the redo-ablation procedure, we first evaluated the patient for the presence of reconnected PVPs in each PV and then conducted the additional ablation.

\section{Analysis of HRV by Holter monitoring}

We analyzed HRV using 24-hour Holter monitor readings obtained at 3-month post-ablation periods using a GE Marquette MARS 8000 Holter analyzer (GE Medical System, Milwaukee, WI, USA). We excluded patients whose HRV was not analyzable because of sinus node dysfunction or a high number of AF $(>1$ hour or $>20$ times per day) or other arrhythmia episodes. After identifying each QRS complex, the numerical series of RR intervals was calculated. Only high-quality recordings were considered for analysis. All recordings were digitized and reviewed by an experienced operator. Premature ventricular beats, premature atrial beats, and electrical artifacts were excluded from analysis. HRV parameters were used as indicators of autonomic activity according to previously published guidelines..$^{10}$ Mean heart rate (beats per min) and certain time-domain HRV parameters were analyzed: mean RR interval (mean NN interval), standard deviation (SD) of NN intervals (SDNN), standard deviation of 5-min means of $\mathrm{NN}$ intervals (SDANN), root-mean square of differences between successive NN intervals (rMSSD), and proportion of adjacent $\mathrm{NN}$ intervals differing by $>50 \mathrm{msec}$ (\%) (pNN50). Some additional parameters were calculated: verylow-frequency components (VLF; <0.04 Hz), low-frequency components (LF; 0.04-0.15 Hz), high-frequency components (HF; $0.15-0.40 \mathrm{~Hz}$ ), and the LF/HF ratio.

\section{Statistical analysis}

Normally distributed continuous variables were expressed as means \pm SDs. ENDU-abl group was matched in a 1:1 ratio by age, sex, and follow-up duration to CONV-abl group. Statistical significance of the comparisons was assessed using either the Student's t-test or Chi-squared test. Kaplan-Meier and Cox regression analyses were used to analyze AF-free survival after catheter ablation. A $p$-value $<0.05$ was considered statistically significant. Statistical analysis was performed using SPSS (version 23.0, Statistical Package for Social Sciences, Chicago, IL, USA) software for Windows.

\section{Results}

Baseline characteristics of the endurance ablation group vs. the conventional ablation group

The clinical characteristics of all 508 patients $(76.0 \%$ men; mean age, $56.2 \pm 9.5$ years) with PAF who were included in this study are summarized in Table 1 . The $\mathrm{CHA}_{2} \mathrm{DS}_{2}-\mathrm{VASc}$ score was $1.3 \pm 1.3$, and the mean LA diameter was $39.8 \pm 5.5 \mathrm{~mm}$. All patients underwent the same ablation lesion set (bidirectional blocks of CPVI and cavotricuspid isthmus ablation). Baseline characteristics of ENDU-abl $(n=254)$ group were similar to those of age-, sex-, and follow-up duration-matched CONV-abl $(n=254)$ group, except for the use of beta-blockers and statins $(p<0.05)$.

Endurance ablation with moderately increased RF power facilitated autonomic modulation and better clinical outcomes

The total procedure time $(181.3 \pm 35.3 \mathrm{~min}$ vs. $163.1 \pm 39.1$ $\min , p<0.001)$ and ablation time $(77.8 \pm 18.1 \mathrm{~min}$ vs. $66.3 \pm 18.6$ min, $p<0.001$ ) were significantly longer in the ENDU-abl than in the CONV-abl group (Table 2). Although procedure-related complication rates were different between the two groups $(6.3 \%$ vs. $1.6 \%, p=0.010$ ), no significant differences were found in terms of major complications between the two groups ( $p=0.088$, Supplementary Table 1). Figure 1 compares representative 3D-electroanatomical maps with ablation marks and VISITAGTM module image (Johnson \& Johnson Inc., Diamond Bar, CA, USA), which represents the stability of 
Table 1. Baseline characteristics

\begin{tabular}{|c|c|c|c|c|}
\hline & $\begin{array}{l}\text { Overall } \\
(\mathrm{N}=508)\end{array}$ & $\begin{array}{l}\text { Endurance ablation } \\
\qquad(\mathrm{N}=254)\end{array}$ & $\begin{array}{l}\text { Conventional ablation } \\
(\mathrm{N}=254)\end{array}$ & $p$-value \\
\hline Age, years & $56.2 \pm 9.5$ & $56.2 \pm 7.1$ & $56.2 \pm 11.4$ & 0.981 \\
\hline Male, n (\%) & $386(76.0)$ & $193(76.0)$ & $193(76.0)$ & $>0.999$ \\
\hline $\mathrm{BSA}, \mathrm{m}^{2}$ & $1.8 \pm 0.2$ & $1.8 \pm 0.2$ & $1.8 \pm 0.2$ & 0.831 \\
\hline $\mathrm{BMI}, \mathrm{kg} / \mathrm{m}^{2}$ & $24.8 \pm 2.9$ & $24.7 \pm 2.9$ & $24.9 \pm 2.9$ & 0.537 \\
\hline \multicolumn{5}{|l|}{ Comorbidities } \\
\hline Heart failure, n (\%) & $20(3.9)$ & $14(5.5)$ & $6(2.4)$ & 0.068 \\
\hline Hypertension, $n(\%)$ & $210(41.3)$ & $102(40.2)$ & $108(42.5)$ & 0.589 \\
\hline Diabetes, n (\%) & $54(10.6)$ & $31(12.2)$ & $23(9.1)$ & 0.249 \\
\hline Stroke or TIA, n (\%) & $41(8.1)$ & $23(9.1)$ & $18(7.1)$ & 0.415 \\
\hline Vascular disease, n (\%) & $68(13.3)$ & $38(15.0)$ & $30(11.8)$ & 0.297 \\
\hline $\mathrm{CHA}_{2} \mathrm{DS}_{2}$-VASc score & $1.3 \pm 1.3$ & $1.3 \pm 1.3$ & $1.3 \pm 1.3$ & 0.706 \\
\hline \multicolumn{5}{|l|}{ Medication } \\
\hline ACE inhibitor or ARB, n (\%) & $158(31.1)$ & $79(31.1)$ & $79(31.1)$ & $>0.999$ \\
\hline Beta-blocker, n (\%) & $174(34.3)$ & $71(28.0)$ & $103(40.6)$ & 0.003 \\
\hline Statin, $n(\%)$ & $134(26.4)$ & $79(31.1)$ & $55(21.7)$ & 0.016 \\
\hline \multicolumn{5}{|l|}{ Echocardiography } \\
\hline LA dimension, mm & $39.8 \pm 5.5$ & $39.7 \pm 5.2$ & $40.0 \pm 5.9$ & 0.548 \\
\hline $\mathrm{LA}$ volume index, $\mathrm{mL} / \mathrm{m}^{2}$ & $30.9 \pm 10.4$ & $30.6 \pm 10.4$ & $31.3 \pm 10.5$ & 0.416 \\
\hline LV end-diastolic dimension, mm & $49.3 \pm 6.4$ & $49.4 \pm 5.0$ & $49.1 \pm 7.5$ & 0.593 \\
\hline LV ejection fraction, \% & $64.1 \pm 8.0$ & $64.7 \pm 7.7$ & $63.6 \pm 8.2$ & 0.136 \\
\hline $\mathrm{E} / \mathrm{Em}$ & $9.5 \pm 5.2$ & $9.7 \pm 5.3$ & $9.3 \pm 5.0$ & 0.334 \\
\hline \multicolumn{5}{|l|}{ CT / Nav X ${ }^{\circledR}$ (first ablation, n=390) } \\
\hline LA volume/BSA, mL/m² & $74.4 \pm 20.1$ & $74.9 \pm 18.5$ & $73.8 \pm 21.8$ & 0.601 \\
\hline LAA volume/BSA, mL/m² & $6.4 \pm 3.0$ & $6.6 \pm 3.1$ & $6.1 \pm 2.8$ & 0.111 \\
\hline Anterior LA volume/BSA, mL/m² & $43.5 \pm 13.1$ & $43.6 \pm 12.3$ & $43.4 \pm 13.9$ & 0.873 \\
\hline
\end{tabular}

ACE, angiotensin converting enzyme; ARB, angiotensin receptor blocker; BMI, body mass index; BSA, body surface area; CT, computed tomography; E/ Em, early mitral inflow velocity to early diastolic mitral annular velocity ratio; LA, left atrium; LAA, left atrial appendage; LV, left ventricle; TIA, transient ischemic attack

ablation catheter maintained longer than $20 \mathrm{~s}$ in each location that ranged $5 \mathrm{~mm}$ diameter. Although ablation marks show CPVIs without gaps in both cases, there are multiple locations with catheter stability $(<20 \mathrm{~s})$ within a $5 \mathrm{~mm}$ diameter range in VISITAG $^{\mathrm{TM}}$ module after CONV-abl. At three months after the procedure, HRV data were available for 410 patients (201 from 
Table 2. Comparisons of procedure outcomes between endurance and conventional ablation groups

\begin{tabular}{lccc}
\hline & $\begin{array}{c}\text { Endurance ablation } \\
(\mathrm{N}=254)\end{array}$ & $\begin{array}{c}\text { Conventional ablation } \\
(\mathrm{N}=254)\end{array}$ & $p$-value \\
\hline Ablation time, min & $77.8 \pm 18.1$ & $66.3 \pm 18.6$ & $<0.001$ \\
Procedure time, min & $181.3 \pm 35.3$ & $163.1 \pm 39.1$ & $<0.001$ \\
HRV parameters at 3-month (n=410) & & & $<0.001$ \\
Mean HR, /min & $76.1 \pm 10.8$ & $70.3 \pm 10.7$ & 0.006 \\
LF domain, ms2 & $8.1 \pm 14.8$ & $12.2 \pm 14.3$ & 0.008 \\
HF domain, ms2 & $6.7 \pm 8.3$ & $8.9 \pm 8.3$ & $<0.001$ \\
LF/HF ratio & $1.1 \pm 0.5$ & $1.3 \pm 0.5$ & \\
Changes in 1-year echocardiography (n=284) & & & 0.038 \\
$\Delta$ LA dimension, mm & $-3.0 \pm 4.1$ & $-1.9 \pm 4.9$ & 0.013 \\
$\Delta$ LA volume index, mL/m² & $-6.1 \pm 8.4$ & $-3.3 \pm 9.5$ & 0.097 \\
$\Delta \mathrm{LV}$ end-diastolic dimension, mm & $-0.3 \pm 5.2$ & $1.4 \pm 9.3$ & 0.179 \\
$\Delta \mathrm{LV}$ ejection fraction, \% & $0.5 \pm 7.6$ & $1.8 \pm 7.8$ & 0.009 \\
$\Delta$ E/Em & $0.0 \pm 4.7$ & $1.7 \pm 5.3$ & \\
\hline
\end{tabular}

E/Em, early mitral inflow velocity to early diastolic mitral annular velocity ratio; $H F$, high frequency; HR, heart rate; HRV, heart rate variability; LA, left atrium; $L F$, low frequency; LV, left ventricle

the ENDU-abl group and 209 from the CONV-abl group), and the LF/HF ratio was significantly lower in the ENDU-abl group than in the CONV-abl group ( $1.1 \pm 0.5$ vs. $1.3 \pm 0.5, p<0.001)$, suggesting a more significant autonomic neural remodeling after ENDU-abl (Table 2). In addition, the reduction in LA dimension ( $\Delta$ LA dimension; $-3.0 \pm 4.1$ vs. $-1.9 \pm 4.9 \mathrm{~mm}, p=0.038$ ) and LA volume index ( $\Delta$ LA volume index; $-6.1 \pm 8.4$ vs. $-3.3 \pm 9.5 \mathrm{~mm}$, $p=0.013)$ were more significant in ENDU-abl group than in CONV-abl group on 1-year post-ablation echocardiogram (Table 2). During $37.2 \pm 17.5$ months of follow-up, the clinical recurrence of AF was significantly lower in ENDU-abl group than in CONV-abl group (19.3\% vs. $35.4 \%$, log-rank $p<0.001$; Figure 2 ).

Redo-ablation findings: low PVP recurrence rate after the endurance ablation

Among the 105 patients with PAF who experienced clinical recurrence, 38 (36.2\%) patients underwent redo-ablation (11 in the ENDU-abl group vs. 27 in the CONV-abl group) at 15.1 \pm 12.1 months after the de novo procedure. PVPs were reconnected in 2.4 \pm 1.5 PV per patient. Reconnected PVPs were observed in $96.3 \%$ (26 of 27) patients from the CONV-abl group, and only in 36.4\% ( 4 of 11) patients from the ENDU-abl group with moderately increased RF power $(p<0.001)$. No anatomical preference was detected in recurred PVPs, and reconnected PVPs were found less frequently after the ENDU-abl than after the CONV-abl strategy independent of the PV sites (Figure 3). Table 3 compares eight patients without PVPs with 30 patients with reconnected PVPs at redo-procedure. In multivariate logistic regression analysis for a total of $152 \mathrm{PV}$ (38 patients with redoprocedure $\times 4 \mathrm{PV}$ ), lower body surface area (odds ratio [OR] 0.023, 95\% confidence interval [CI] 0.001-0.595, $p=0.023$ ), smaller LA volume index (CT measured; OR 0.979, 95\% CI $0.960-0.998, p=0.032$ ), and the ENDU-abl strategy (OR 7.715, $95 \%$ CI 2.355-25.273, $p<0.001)$ were independently associated with the absence of PVPs at redo-ablation procedures (Table 4). 


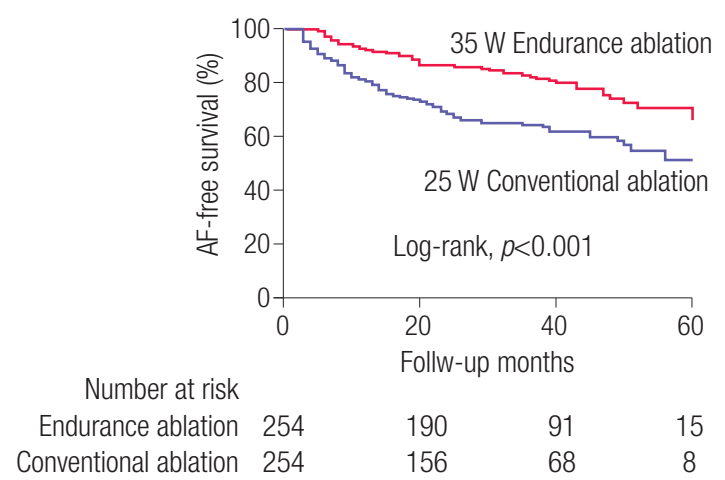

Figure 2. Comparison of AF-free survival in the endurance ablation with moderately increased RF power and conventional ablation groups $A F$, atrial fibrillation; $R F$, radiofrequency

\section{Discussion}

In this study, we compared the outcomes of patients with PAF between the ENDU-abl group (30 s ablation at each ablation point) with those of age-, sex-, and follow-up duration-matched patients from the CONV-abl group (elimination of PVPs only). Despite the longer procedure time, the ENDU-abl with moderately increased RF power resulted in better autonomic neural remodeling as measured using HRV, more significant LA reverse remodeling at 1 -year follow-up echocardiogram, lower clinical recurrence rate of $\mathrm{AF}$, and lower reconnection rate of PVPs at the time of redo-procedure than in patients who underwent CONV-abl. However, procedure-related complication rate was higher after ENDU-abl than after CONVabl without significant increase of major complications rate. We took care to reduce the RF power at the LA posterior wall in both groups.

\section{Catheter ablation for PAF}

Catheter ablation is a well-established procedure for antiarrhythmic drug-resistant PAF, which is considered a class IA indication according to the $2014 \mathrm{ACC} / \mathrm{AHA} / \mathrm{HRS}$ guidelines ${ }^{11}$ and the 2016 ESC guidelines. ${ }^{12}$ Current guidelines even recommend RFCA as the first line therapy at experienced centers,

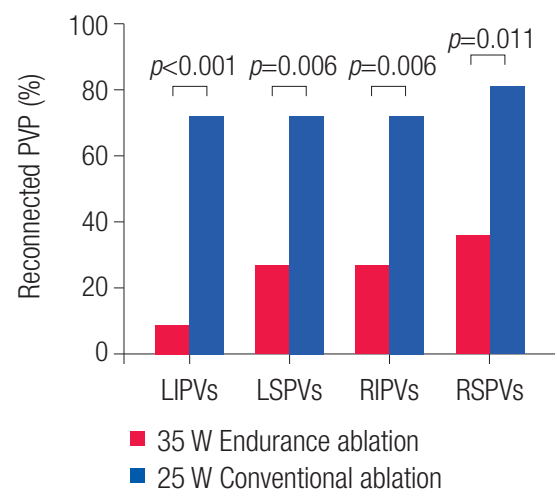

Figure 3. Reconnected PVPs according to the anatomical location of PVS LIPVs, left inferior pulmonary veins; LSPVs, left superior pulmonary veins; PV, pulmonary vein; PVPs, pulmonary vein potentials; RIPVs, right inferior pulmonary veins; RSPVs, right superior pulmonary veins

depending on patient preference. ${ }^{13}$ However, a substantial clinical recurrence rate of AF (28-53\%) persists in reports from worldclass institutes. ${ }^{14}$ This limitation of AF ablation has been overcome in several ways. First, in the last several decades, the efficacy and safety of AF ablation have continuously improved, especially with the development of the irrigated tip catheter and $3 \mathrm{D}$ electroanatomical mapping system. The irrigated tip catheter secured long-lasting autonomic modulation ${ }^{7}$ and improved clinical outcomes. ${ }^{15}$ The $3 \mathrm{D}$ electroanatomical mapping system enabled the high bidirectional block rate of linear ablation and the reproducible complex fractionated atrial electrogram (CFAE) guided ablation. Second, we have come to understand the clinical characteristics of patients with a high chance of recurrence after RFCA, and this has enabled the selection of appropriate patients for catheter ablation. For example, guidelines do not recommend AF ablation in patients with an LA dimension greater than 55 $\mathrm{mm}$, whereas patients with obstructive sleep apnea ${ }^{16}$ or high pericardial fat volume ${ }^{17}$ are vulnerable to post-procedural clinical recurrence. However, AF catheter ablation remains an operatordependent procedure despite improvements in devices and better patient selection. When various CPVI strategies were compared in patients with PAF, neither additional linear ablation ${ }^{18,19}$ nor CFAE ablation ${ }^{20}$ improved clinical outcomes. Therefore, longlasting CPVI might be a cornerstone of PAF ablation, and we demonstrated the superiority of ENDU-abl in terms of 
Table 3. Clinical characteristics depending on the state of the PVP at the time of redo-ablation among 38 PAF patients underwent redo-ablation

\begin{tabular}{|c|c|c|c|}
\hline & No PVP $(\mathrm{N}=8)$ & $P V P+(N=30)$ & $p$-value \\
\hline Age, years & $52.6 \pm 8.1$ & $53.1 \pm 11.5$ & 0.919 \\
\hline Male, n (\%) & $3(37.5)$ & $23(76.7)$ & 0.034 \\
\hline $\mathrm{BSA}, \mathrm{m}^{2}$ & $1.7 \pm 0.1$ & $1.8 \pm 0.2$ & 0.137 \\
\hline $\mathrm{BMI}, \mathrm{kg} / \mathrm{m}^{2}$ & $24.3 \pm 3.2$ & $25.1 \pm 3.0$ & 0.481 \\
\hline Heart failure, n (\%) & $1(12.5)$ & $2(6.7)$ & 0.587 \\
\hline Hypertension, n (\%) & $2(25)$ & $7(23.3)$ & 0.922 \\
\hline Diabetes, n (\%) & 0 & $5(16.7)$ & 0.023 \\
\hline Stroke or TIA, $n(\%)$ & 0 & $1(3.3)$ & 0.601 \\
\hline $\mathrm{CHA}_{2} \mathrm{DS}_{2}$-VASc score & $0.9 \pm 0.8$ & $1.0 \pm 1.0$ & 0.684 \\
\hline \multicolumn{4}{|l|}{ Echocardiography (de-novo) } \\
\hline LA dimension, mm & $38.7 \pm 5.6$ & $38.4 \pm 6.9$ & 0.926 \\
\hline $\mathrm{LA}$ volume index, $\mathrm{mL} / \mathrm{m}^{2}$ & $29.1 \pm 6.7$ & $26.5 \pm 9.1$ & 0.520 \\
\hline \multicolumn{4}{|l|}{ CT and NavX (de-novo) } \\
\hline LA volume/BSA, mL/m² & $63.0 \pm 20.5$ & $82.6 \pm 26.3$ & 0.105 \\
\hline Anterior LA volume/BSA, mL/m² & $34.4 \pm 12.1$ & $49.0 \pm 16.9$ & 0.060 \\
\hline Mean LA voltage, $\mathrm{mV}$ & $1.2 \pm 0.2$ & $0.8 \pm 0.4$ & 0.144 \\
\hline Endurance ablation, n (\%) & $7(87.5)$ & $4(13.3)$ & $<0.001$ \\
\hline Ablation time, min & $75.5 \pm 14.4$ & $71.0 \pm 20.2$ & 0.614 \\
\hline Procedure time, min & $207.7 \pm 50.7$ & $163.2 \pm 38.6$ & 0.024 \\
\hline
\end{tabular}

BMI, body mass index; BSA, body surface area; CT, computed tomography; LA, left atrium; PAF, paroxysmal atrial fibrillation; PVP, pulmonary vein potential; TIA, transient ischemic attack

autonomic modulation, LA reverse remodeling, clinical outcomes, and long-term maintenance of PVI in this study. The elimination of PVPs and PV entrance/exit blocks are the most widely accepted endpoints and minimum criteria for electrical isolation. However, the ENDU-abl strategy, which was maintained for at least $30 \mathrm{~s}$ at target points, enabled sufficient transmural lesion formation in addition to eliminating dormant PVP. Ouyang et al..$^{21}$ reported that the PV reconnection rate was very high $(94.0 \%)$ in their analysis of 66 patients who underwent redo-ablation for symptomatic PAF, even when using a maximal power of $40 \mathrm{~W}$. This result suggests that ENDU-abl strategy at the target site is important, as is the use of high power ablation. While transmural lesion formation is important, there is a potential risk of collateral damage. Although the ENDU-abl with moderately increased RF power had an acceptable cardiac complication rate when compared to CONV-abl, it is essential to avoid potential complications, especially at the LA posterior wall, which is close to the esophagus. 
Table 3. Predictors of the absence of PVPs at the time of redo-ablation (38 patients $\times 4$ pulmonary veins $=152$ PVs)

\begin{tabular}{|c|c|c|c|c|c|c|}
\hline & \multicolumn{3}{|c|}{ Univariate analysis } & \multicolumn{3}{|c|}{ Multivariate analysis } \\
\hline & $\mathrm{OR}$ & $95 \% \mathrm{Cl}$ & $p$-value & $\mathrm{OR}$ & $95 \% \mathrm{Cl}$ & $p$-value \\
\hline Age & 1.000 & $0.970-1.031$ & 0.994 & & & \\
\hline Male sex & 0.290 & $0.142-0.591$ & 0.001 & 0.602 & $0.174-2.083$ & 0.423 \\
\hline BSA & 0.031 & $0.003-0.350$ & 0.005 & 0.023 & $0.001-0.595$ & 0.023 \\
\hline BMI & 0.886 & $0.781-1.006$ & 0.061 & & & \\
\hline Heart failure & 2.413 & $0.725-8.031$ & 0.151 & & & \\
\hline Hypertension & 0.738 & $0.333-1.636$ & 0.455 & & & \\
\hline Diabetes & 0.198 & $0.143-0.908$ & 0.037 & 0.311 & $0.057-1.692$ & 0.177 \\
\hline Stroke or TIA & 0.522 & $0.053-5.151$ & 0.578 & & & \\
\hline $\mathrm{CHA}_{2} \mathrm{DS}_{2}$-VASc score & 0.912 & $0.598-1.392$ & 0.669 & & & \\
\hline LA dimension (Echo) & 0.976 & $0.922-1.033$ & 0.402 & & & \\
\hline LA volume index (Echo) & 1.006 & $0.963-1.050$ & 0.800 & & & \\
\hline LA volume/BSA (CT) & 0.979 & $0.963-0.995$ & 0.010 & 0.979 & $0.960-0.998$ & 0.032 \\
\hline Anterior LA volume/BSA (CT) & 0.961 & $0.937-0.986$ & 0.003 & & & \\
\hline Ablation time (de-novo) & 1.000 & $1.000-1.001$ & 0.130 & & & \\
\hline Endurance ablation & 9.960 & $4.405-22.520$ & $<0.001$ & 7.715 & $2.355-25.273$ & 0.001 \\
\hline
\end{tabular}

BMI, body mass index; BSA, body surface area; Cl, confidence interval; CT, computed tomography; Echo, echocardiogram; LA, left atrium; OR, odds ratio; PV, pulmonary vein; PVP, pulmonary vein potential; TIA, transient ischemic attack

Long-lasting CPVI and autonomic neural remodeling

The cardiac autonomic nerves play an important role in initiation and maintenance of arrhythmias, and vagal suppression is known to improve the clinical outcome of AF ablation.22 Cardiac autonomic nerves and ganglionated plexi are located along the epicardial aspect of the PV antrum. Therefore, the ENDU-abl of PV promotes transmural lesion formation and long-lasting autonomic neural remodeling. ${ }^{7}$ In addition, a reduction in LF/HF ratio in follow-up heart rate variability analysis is an independent predictor of AF recurrence after RFCA. 7 In this study, lower PVP recurrence during the redoprocedure and more significant reduction of the follow-up LF/ HF ratio were indirect evidences of transmural lesion formation through the ENDU-abl compared to elimination of PVP alone
(CONV-abl). Therefore, CPVI may not be an ultimate goal of PAF ablation, but an indicator for antral endocardial lesion formation or a minimum requirement to block PV triggers. However, even though $63 \%$ of patients from the ENDU-abl group who underwent a redo-procedure did not show the reconnection of PVPs, PAF recurred. This finding indicates that there is still a high frequency of non-PV foci in patients with recurred PAF after the ENDU-abl with moderately increased RF power.

Potential complications and avoidance of esophageal injury

It is essential to avoid collateral damage during the ENDU-abl for PAF. We evaluated eight cases of hemopericardium and two 
cases of pericarditis in the ENDU-abl group. All cases of hemopericardium were controlled via percutaneous pericardial drainage. In addition, high RF power ablation with coaxial contact force carries a risk of cardiac perforation, especially at the roof of the PV antrum. The most dangerous potential complication is esophageal injury. To avoid this fatal complication, we were careful to reduce the RF power to $25-30 \mathrm{~W}$; moreover, we reduced the depth of conscious sedation when the ablation line passed through the posterior wall of LA. If the patient complained of pain or voltage abatement was observed, we moved the ablation catheter without waiting for $30 \mathrm{~s}$ even in the ENDU-abl group.

\section{Limitations}

Our study had several limitations. First, the study design was retrospective. The choice of the ablation strategy depended on the operators' discretion and experience. To overcome this limitation, we selected the subjects of the CONV-abl group through age-, sex-, and follow-up duration-matching with those of the ENDUabl group. Second, this study included a highly selected group of patients with PAF who were referred for RFCA. Therefore, the results of this study cannot be generalized. Third, patients who used a contact force-sensing ablation catheter were not included in the current study design; therefore, the information on the forcetime integral for CPVI could not be compared between the two groups. Fourth, HRV parameters estimate relative changes in sympathovagal activities, and HRV analysis requires normal sinus rhythm with normal cardiac function; thus, we excluded patients whose Holter ECGs could not be analyzed for HRV.

\section{Conclusion}

Despite the longer procedure time, the endurance ablation technique with moderately increased RF power resulted in better autonomic neural remodeling as measured using HRV, more significant reverse remodeling of the LA, lower clinical recurrence rate of $\mathrm{AF}$, and lower reconnection rate of PVPs at the time of the redo-procedure compared to conventional ablation. Moreover, these benefits were achieved without significant increase of major complications rate. However, when using this procedure, it is essential to avoid potential collateral damage, especially at the LA posterior wall near the esophagus.

\section{Acknowledgements}

We thank Mr. Jungkee Lee for his technical support in electroanatomical mapping.

\section{Funding}

This study was supported by a research grant from the Korean Heart Rhythm Society (KHRS 2016-4).

\section{Disclosures}

The authors declare that there is no conflict of interest.

\section{References}

1) Nademanee K, McKenzie J, Kosar E, Schwab M, Sunsaneewitayakul B, Vasavakul T, Khunnawat C, Ngarmukos T. A new approach for catheter ablation of atrial fibrillation: mapping of the electrophysiologic substrate. J Am Coll Cardiol. 2004;43:20442053.

2) Knecht S, Hocini M, Wright M, Lellouche N, O'Neill MD, Matsuo S, Nault I, Chauhan VS, Makati KJ, Bevilacqua M, Lim KT, Sacher F, Deplagne A, Derval N, Bordachar P, Jaïs P, Clémenty J, Haïssaguerre M. Left atrial linear lesions are required for successful treatment of persistent atrial fibrillation. Eur Heart J. 2008;29:23592366.

3) Oral H, Knight BP, Tada H, Ozaydin M, Chugh A, Hassan S, Scharf C, Lai SW, Greenstein R, Pelosi F Jr, Strickberger SA, Morady F. Pulmonary vein isolation for paroxysmal and persistent atrial fibrillation. Circulation. 2002;105:1077-1081.

4) Cappato R, Negroni S, Pecora D, Bentivegna S, Lupo PP, Carolei A, Esposito C, Furlanello F, De Ambroggi L. Prospective assessment of late conduction recurrence across radiofrequency lesions producing electrical disconnection at the pulmonary vein ostium in patients with atrial fibrillation. Circulation. 2003;108:1599-1604.

5) Hwang ES, Nam GB, Joung B, Park J, Lee JS, Shim J, Uhm JS, Lee MH, Pak HN. Significant reduction of atrial defibrillation threshold and inducibility by catheter ablation of atrial fibrillation. 


\section{Pacing Clin Electrophysiol. 2012;35:1428-1435.}

6) Shim J, Joung B, Park JH, Uhm JS, Lee MH, Pak HN. Long duration of radiofrequency energy delivery is an independent predictor of clinical recurrence after catheter ablation of atrial fibrillation: over 500 cases experience. Int J Cardiol. 2013;167:26672672.

7) Kang KW, Kim TH, Park J, Uhm JS, Joung B, Hwang C, Lee MH, Pak HN. Long-term changes in heart rate variability after radiofrequency catheter ablation for atrial fibrillation: 1-year followup study with irrigation tip catheter. J Cardiovasc Electrophysiol. 2014;25:693-700.

8) Lang RM, Badano LP, Mor-Avi V, Afilalo J, Armstrong A, Ernande L, Flachskampf FA, Foster E, Goldstein SA, Kuznetsova T, Lancellotti P, Muraru D, Picard MH, Rietzschel ER, Rudski L, Spencer KT, Tsang W, Voigt JU. Recommendations for cardiac chamber quantification by echocardiography in adults: an update from the American Society of Echocardiography and the European Association of Cardiovascular Imaging. J Am Soc Echocardiogr. 2015;28:1-39 e14.

9) European Heart Rhythm Association (EHRA); European Cardiac Arrhythmia Scoiety (ECAS); American College of Cardiology (ACC); American Heart Association (AHA); Society of Thoracic Surgeons (STS), Calkins H, Brugada J, Packer DL, Cappato R, Chen SA, Crijns HJ, Damiano RJ Jr, Davies DW, Haines DE, Haissaguerre M, Iesaka Y, Jackman W, Jais P, Kottkamp H, Kuck KH, Lindsay BD, Marchlinski FE, McCarthy PM, Mont JL, Morady F, Nademanee K, Natale A, Pappone C, Prystowsky E, Raviele A, Ruskin JN, Shemin RJ. HRS/EHRA/ECAS expert Consensus Statement on catheter and surgical ablation of atrial fibrillation: recommendations for personnel, policy, procedures and follow-up. a report of the Heart Rhythm Society (HRS) Task Force on catheter and surgical ablation of atrial fibrillation. Heart Rhythm. 2007;4:816-861.

10) Heart rate variability: standards of measurement, physiological interpretation and clinical use. Task Force of the European Society of Cardiology and the North American Society of Pacing and Electrophysiology. Circulation. 1996;93:1043-1065.

11) January CT, Wann LS, Alpert JS, Calkins H, Cigarroa JE, Cleveland JC, Jr., Conti JB, Ellinor PT, Ezekowitz MD, Field ME, Murray KT, Sacco RL, Stevenson WG, Tchou PJ, Tracy CM, Yancy CW; ACC/AHA Task Force Members. 2014 AHA/ ACC/HRS guideline for the management of patients with atrial fibrillation: a report of the American College of Cardiology/
American Heart Association Task Force on practice guidelines and the Heart Rhythm Society. Circulation. 2014;130:e199-267.

12) Kirchhof P, Benussi S, Kotecha D, Ahlsson A, Atar D, Casadei B, Castella M, Diener HC, Heidbuchel H, Hendriks J, Hindricks G, Manolis AS, Oldgren J, Popescu BA, Schotten U, Van Putte B, Vardas P, Agewall S, Camm J, Baron Esquivias G, Budts W, Carerj S, Casselman F, Coca A, De Caterina R, Deftereos S, Dobrev D, Ferro JM, Filippatos G, Fitzsimons D, Gorenek B1, Guenoun M, Hohnloser SH, Kolh P, Lip GY, Manolis A, McMurray J, Ponikowski P, Rosenhek R, Ruschitzka F, Savelieva I, Sharma S, Suwalski P, Tamargo JL, Taylor CJ, Van Gelder IC, Voors AA, Windecker S, Zamorano JL, Zeppenfeld K. 2016 ESC Guidelines for the management of atrial fibrillation developed in collaboration with EACTS. Eur Heart J. 2016;37:2893-2962.

13) Morillo CA, Verma A, Connolly SJ, Kuck KH, Nair GM, Champagne J, Sterns LD, Beresh H, Healey JS, Natale A; RAAFT-2 Investigators. Radiofrequency ablation vs antiarrhythmic drugs as first-line treatment of paroxysmal atrial fibrillation (RAAFT-2): a randomized trial. JAMA. 2014;311:692700.

14) Brooks AG, Stiles MK, Laborderie J, Lau DH, Kuklik P, Shipp NJ, Hsu LF, Sanders P. Outcomes of long-standing persistent atrial fibrillation ablation: a systematic review. Heart Rhythm. 2010;7:835-846.

15) Hwang ES, Pak HN, Park SW, Park JS, Joung B, Choi D, Lee $\mathrm{MH}, \mathrm{Kim} \mathrm{YH}$. Risks and benefits of an open irrigation tip catheter in intensive radiofrequency catheter ablation in patients with nonparoxysmal atrial fibrillation. Circ J. 2010;74:644-649.

16) Fein AS, Shvilkin A, Shah D, Haffajee CI, Das S, Kumar K, Kramer DB, Zimetbaum PJ, Buxton AE, Josephson ME, Anter E. Treatment of obstructive sleep apnea reduces the risk of atrial fibrillation recurrence after catheter ablation. J Am Coll Cardiol. 2013;62:300-305.

17) Kim TH, Park J, Park JK, Uhm JS, Joung B, Lee MH, Pak HN. Pericardial fat volume is associated with clinical recurrence after catheter ablation for persistent atrial fibrillation, but not paroxysmal atrial fibrillation: An analysis of over 600-patients. Int J Cardiol. 2014; 176:841-846.

18) Mun HS, Joung B, Shim J, Hwang HJ, Kim JY, Lee MH, Pak HN. Does additional linear ablation after circumferential pulmonary vein isolation improve clinical outcome in patients with paroxysmal atrial fibrillation? Prospective randomised study. 
Heart. 2012;98:480-484.

19) Kim TH, Park J, Park JK, Uhm JS, Joung B, Hwang C, Lee MH, Pak HN. Linear ablation in addition to circumferential pulmonary vein isolation (Dallas lesion set) does not improve clinical outcome in patients with paroxysmal atrial fibrillation: a prospective randomized study. Europace. 2015;17:388-395.

20) Verma A, Mantovan R, Macle L, De Martino G, Chen J, Morillo CA, Novak P, Calzolari V, Guerra PG, Nair G, Torrecilla EG, Khaykin Y. Substrate and Trigger Ablation for Reduction of Atrial Fibrillation (STAR AF): a randomized, multicentre, international trial. Eur Heart J. 2010;31:1344-1356.
21) Ouyang F, Tilz R, Chun J, Schmidt B, Wissner E, Zerm T, Neven K, Köktürk B, Konstantinidou M, Metzner A, Fuernkranz A, Kuck KH. Long-term results of catheter ablation in paroxysmal atrial fibrillation: lessons from a 5-year follow-up. Circulation. 2010;122:2368-2377.

22) Pappone C, Santinelli V, Manguso F, Vicedomini G, Gugliotta F, Augello G, Mazzone P, Tortoriello V, Landoni G, Zangrillo A, Lang C, Tomita T, Mesas C, Mastella E, Alfieri O. Pulmonary vein denervation enhances long-term benefit after circumferential ablation for paroxysmal atrial fibrillation. Circulation. 2004;109:327-334. 